\title{
ON CERTAIN DISTRIBUTIONS OF INTEGERS IN PAIRS WITH GIVEN DIFFERENCES
}

\author{
TH. SKOLEM
}

1. A study of the structure of some triple systems of Steiner (cf. [2]) led me to consider the following problem: Is it possible to distribute the numbers $1,2, \ldots, 2 n$ in $n$ pairs $\left(a_{r}, b_{r}\right)$ such that we have $b_{r}-a_{r}=r$ for $r=1,2, \ldots, n$ ?

In the sequel, a set of pairs of this kind is called a $1,+1$ system because the differences $b_{r}-a_{r}$ begin with 1 and increase by 1 when $r$ increases by 1 . One finds very soon that such a system does not always exist. In the simplest case $n=1$ we have only the two numbers 1,2 which quite trivially form a pair that is a system of the kind considered. But already in the case $n=2$ there is no $1,+1$ system. Indeed the only distributions of $1,2,3,4$ in two pairs are

$$
(1,2)(3,4) \quad(1,3)(2,4) \quad(2,3)(1,4)
$$

with the corresponding differences

$$
1,1 \quad 2,2 \quad 1,3
$$

so that we never have just the differences 1,2 .

Also for $n=3$ one easily finds that no $1,+1$ system exists. However, for $n=4$ there is again such a system, namely

$$
(6,7)(1,3)(2,5)(4,8) \text {. }
$$

Thus the question arises: For which $n$ does a $1,+1$ system of pairs exist? The complete answer is given by the two theorems:

Theorem 1. If $n \equiv 2$ or $3(\bmod 4)$, no $1,+1$ system exists.

Theorem 2. If $n \equiv 0$ or $1(\bmod 4)$, then $a 1,+1$ system always exists.

Proof of Theorem 1. I give here a very short proof due to Professor Th. Bang, my own original proof being somewhat longer. If the pairs 
$\left(a_{r}, b_{r}\right), r=1, \ldots, n$, constitute a $1,+1$ system of the numbers $1, \ldots, 2 n$, then we have the equations

$$
b_{r}-a_{r}=r, \quad r=1,2, \ldots, n,
$$

whence by summation

$$
\Sigma b_{r}-\Sigma a_{r}=\frac{1}{2} n(n+1) .
$$

On the other hand, since the collection of the numbers $a_{r}$ and $b_{r}$ is the set $1,2, \ldots, 2 n$, we also have

$$
\Sigma b_{r}+\Sigma a_{r}=n(2 n+1) .
$$

Addition of the two equations yields

$$
\Sigma b_{r}=\frac{1}{4} n(5 n+3),
$$

which is an integer only when $n \equiv 0$ or $1(\bmod 4)$.

Proof of Theorem 2. Let first $n \equiv 0(\bmod 4)$. It will then suffice to give a general description of a $1,+1$ system for an arbitrary $n=4 m$. Such a description is the following: The system of pairs consists of

1) all pairs $(4 m+r, 8 m-r)$ for $r=0,1, \ldots, 2 m-1$,

2) the pairs $(2 m+1,6 m)$ and $(2 m, 4 m-1)$,

$3)$ the pairs $(r, 4 m-1-r)$ for $r=1,2, \ldots, m-1$,

4) the pair $(m, m+1)$,

5) the pairs $(m+2+r, 3 m-1-r)$ for $r=0,1, \ldots, m-3$.

The pairs 1) give all the even differences $2,4, \ldots, 4 m$. The two odd differences $2 m-1$ and $4 m-1$ are obtained from 2). The least difference 1 is got from 4 ), the differences $3,5, \ldots, 2 m-3$ from 5 ) and the remaining odd differences $2 m+1, \ldots, 4 m-3$ from 3 ).

Now let $n \equiv 1(\bmod 4)$. As pointed out by Professor Bang it is possible also in this case to give a general description of a $1,+1$ system which is quite analogous to that given by me above for the case $n \equiv 0(\bmod 4)$. Indeed, setting $n=4 m+1$, the system consists of

1) all pairs $(4 m+2+r, 8 m+2-r)$ for $r=0,1, \ldots, 2 m-1$,

2) the pairs $(2 m+1,6 m+2)$ and $(2 m+2,4 m+1)$,

$3)$ the pairs $(r, 4 m+1-r)$ for $r=1,2, \ldots, m$,

4) the pair $(m+1, m+2)$,

5) the pairs $(m+2+r, 3 m+1-r)$ for $r=1,2, \ldots, m-2$.

The pairs 1) give all even differences $2,4, \ldots, 4 m$. The two odd differences $2 m-1$ and $4 m+1$ are given by 2). The least difference 1 is obtained from 4), the differences $3,5, \ldots, 2 m-3$ from 5 ), and the odd differences $2 m+1, \ldots, 4 m-1$ from 3 ). 
In the cases $n \equiv 0,1(\bmod 4)$ the number of $1,+1$ systems of pairs built from the integers $1, \ldots, 2 n$ will probably increase indefinitely when $n$ increases to infinity, but $I$ will not here make any attempt to treat this question.

2. I shall now make some remarks concerning the extension of this problem to the whole number series. It is clear that in this case the existence of a $1,+1$ system is quite trivial. More generally it is obvious that $l,+m$ systems exist, that means systems of disjoint pairs such that the corresponding differences are the numbers $l, l+m, l+2 m, \ldots$. The reason for my treatment of these systems is that some quite peculiar theorems may be proved in this connection.

The simplest procedure for constructing a $1,+1$ system of all the integers is as follows. The first pair may be $\left(a_{1}, b_{1}\right)$, where $a_{1}$ is 1 and $b_{1}$ is 2 . Then the $n$th pair $\left(a_{n}, b_{n}\right)$ is built by recursion by letting $a_{n}$ be the least integer which is different from all $a_{r}$ and $b_{r}$ for $r=1,2, \ldots, n-1$ and setting $b_{n}=a_{n}+n$. I list here the first 29 of these pairs:

$$
\begin{aligned}
& (1,2)(3,5)(4,7)(6,10)(8,13)(9,15)(11,18)(12,20)(14,23) \\
& (16,26)(17,28)(19,31)(21,34)(22,36)(24,39)(25,41)(27,44) \\
& (29,47)(30,49)(32,52)(33,54)(35,57)(37,60)(38,62)(40,65) \\
& (42,68)(43,70)(45,73)(46,75) \text {. }
\end{aligned}
$$

I was a little surprised when I discovered that these pairs can be given by a simple formula. Indeed we have

$$
a_{n}=\left[\frac{1}{2}\left(1+5^{\frac{1}{2}}\right) n\right], \quad b_{n}=\left[\frac{1}{2}\left(3+5^{\frac{1}{2}}\right) n\right],
$$

where $[\xi]$ as usual denotes the greatest integer $\leqq \xi$. This will be proved in Theorem $\mathbf{3 a}$.

I shall first prove some other statements. Let $\alpha$ be the positive root of the equation

$$
\alpha^{2}-\alpha-1=0,
$$

thus $\alpha=\frac{1}{2}\left(1+5^{\frac{1}{2}}\right), \alpha^{2}=\frac{1}{2}\left(3+5^{\frac{1}{2}}\right)$. Then the propositions are:

1. If $n=[\alpha m]$, then $[\alpha n]=\left[\alpha^{2} m\right]-1$.

2. If $n=[\alpha m]+1$, then $[\alpha n]=\left[\alpha^{2} m\right]+1$.

3. If $n=[\alpha m]$, then $[\alpha(n+1)]=[\alpha n]+2$.

4. If $n=[\alpha m]+1$, while $[\alpha(m+1)]=[\alpha m]+2$, then $[\alpha(n+1)]=[\alpha n]+1$.

Proof of 1. From $n=[\alpha m]$ it follows that

$$
n^{2}-m n-m^{2}<0, \quad(n+1)^{2}-m(n+1)-m^{2}>0,
$$

where the inequality to the right may be written 
Hence

$$
n^{2}-m n-m^{2}-m+2 n+1>0 \text {. }
$$

$$
(n+m-1)^{2}-n(n+m-1)-n^{2}=-n^{2}+m n+m^{2}-2 m-n+1<0,
$$

because the left-hand side equals $-n^{2}+m n+m^{2}+m-2 n-1-3 m+n+2$, and $-n^{2}+m n+m^{2}+m-2 n-1$ and $-3 m+n+2$ are both negative. Further

$$
(n+m)^{2}-n(n+m)-n^{2}=-n^{2}+m n+m^{2}>0 .
$$

Thus it is proved that

$$
n+m-1=\left[\alpha^{2} m\right]-1=[\alpha n] .
$$

Proof of 2. From $n-1=[\alpha m]$ it follows that

$$
(n-1)^{2}-m(n-1)-m^{2}<0, \quad n^{2}-m n-m^{2}>0 .
$$

The inequality to the left is

$$
n^{2}-m n-m^{2}+m-2 n+1<0 .
$$

We have

$$
(n+m)^{2}-n(n+m)-n^{2}=-n^{2}+m n+m^{2}<0 .
$$

Further

$$
\begin{aligned}
(n+m+1)^{2}-n(n+m+1)-n^{2} & =-n^{2}+m n+m^{2}+2 m+n+1 \\
& =-n^{2}+m n+m^{2}-m+2 n-1+3 m-n+2,
\end{aligned}
$$

which is positive. Thus we have proved that

$$
n+m=\left[\alpha^{2} m\right]+1=[\alpha n] .
$$

Proof of 3. The statement may be written

$$
\left[\alpha^{2} m\right]+1=[\alpha(n+1)]
$$

under the same hypothesis as in proposition 1. Now

$$
(n+m+1)^{2}-(n+1)(n+m+1)-(n+1)^{2}=-n^{2}+m n+m^{2}+m-2 n-1,
$$

which (see the proof of 1.) is negative. On the other hand

$$
(n+m+2)^{2}-(n+1)(n+m+2)-(n+1)^{2}=-n^{2}+m n+m^{2}+3 m-n+1,
$$

which is positive because $-n^{2}+m n+m^{2}>0$ and $3 m-n+1>0$. Thus

$$
n+m+1=\left[\alpha^{2} m\right]+1=[\alpha(n+1)] .
$$

Proof of 4. The statement may be written

$$
\left[\alpha^{2} m\right]+2=[\alpha(n+1)] .
$$


We have the two inequalities expressing the hypothesis of proposition 2 and further

that is,

$$
\begin{aligned}
& (n+1)^{2}-(m+1)(n+1)-(m+1)^{2}<0, \\
& (n+2)^{2}-(m+1)(n+2)-(m+1)^{2}>0,
\end{aligned}
$$

$$
n^{2}-m n-m^{2}-3 m+n-1<0, \quad n^{2}-m n-m^{2}-4 m+3 n+1>0 .
$$

Now we have

$$
\begin{aligned}
(n+m+1)^{2}-(n+1)(n+m+1) & -(n+1)^{2} \\
& =-n^{2}+m n+m^{2}+m-2 n-1<0
\end{aligned}
$$

because $-n^{2}+m n+m^{2}<0$ (see the proof of 2 .) and $m-2 n-1<0$. Further,

Thus

$$
\begin{aligned}
(n+m+2)^{2}-(n+1)(n+m+2) & -(n+1)^{2} \\
& =-n^{2}+m n+m^{2}+3 m-n+1>0 .
\end{aligned}
$$

$$
n+m+1=\left[\alpha^{2} m\right]+2=[\alpha(n+1)] .
$$

It is now easy to prove the following theorem:

TheOREM 3 a. Every positive integer is of one and only one of the two forms

$$
[\alpha n], \quad\left[\alpha^{2} n\right],
$$

where $n$ denotes some positive integer. Further the pairs obtained by the procedure explained above are just the pairs $\left([\alpha n],\left[\alpha^{2} n\right]\right)$.

Proof. My first proof of this theorem was based on the preceding four lemmas. However, a reproduction of this proof here is superfluous because it is easily verified that the second statement in Theorem $3 \mathrm{a}$ is a special case of Theorem 4, which is proved below. Then the first proposition in Theorem $3 \mathrm{a}$ is proved by the following simple argument: Since every integer is of one of the two forms $[\alpha n]$ or $\left[\alpha^{2} n\right]$, the least integer which does not belong to any of the pairs $\left([\alpha r],\left[\alpha^{2} r\right]\right)$, $r=1,2, \ldots, n-1$, must occur as the least integer in the pairs ( $\left.[\alpha s],\left[\alpha^{2} s\right]\right)$ for $s=n, n+1, \ldots$. It is then evident that $a_{n}$ is just this number, which means that the pairs obtained by the recursive procedure explained above are just the pairs $\left([\alpha n],\left[\alpha^{2} n\right]\right)$.

A more general theorem is:

TheORem $3 \mathrm{~b}$. Let $m$ be an arbitrary natural number and $l$ one of the numbers $1, \ldots m$. Further let $N_{1}$ be the set of integers of the form

$$
f(n)=\left[\frac{1}{2}\left(2-m+\left(m^{2}+4\right)^{\frac{1}{2}}\right)\left(n-\frac{m-l}{m}\right)+\frac{2(m-l)}{m}\right]
$$


and $N_{2}$ the set of integers of the form

$$
g(n)=\left[\frac{1}{2}\left(2+m+\left(m^{2}+4\right)^{\frac{1}{2}}\right)\left(n-\frac{m-l}{m}\right)+\frac{2(m-l)}{m}\right] .
$$

Then $N_{1}$ and $N_{2}$ are complementary subsets of the natural number series $N$ and the pairs $(f(n), g(n))$ constitute $a l,+m$ system.

I omit the proof, which can be performed by considerations analogous to those in the proof of Theorem 3 a.

3. The relation between two sets of the forms $[\alpha n]$ and $[\beta n]$ may be very different in different cases. I should like to give an example, where one of the two sets is contained in the other. Of course this phenomenon is trivial in the case that $\alpha / \beta$ or $\beta / \alpha$ is an integer. It is worth noticing, however, that it can also happen when $\alpha / \beta$ is irrational, which is shown by the following example: Every integer of the form $\left[\left(1+2^{\frac{1}{2}}\right) n\right]$ is also of the form $\left[2^{\frac{1}{2}} n\right]$. Indeed, I shall prove the general validity of the formula

where

$$
\left[\left(1+2^{\frac{1}{2}}\right) m\right]=\left[2^{\frac{1}{2}} l\right],
$$

$$
l=\left[\left(2^{-\frac{1}{2}}+1\right) m+\frac{1}{2}\right],
$$

so that $l$ is the integer nearest to $\left(2^{-\frac{1}{2}}+1\right) m$.

Let $n=\left[2^{\frac{1}{2}} m\right]$, so that we have

Then

$$
2^{\frac{1}{2}} m=n+\varepsilon, \quad 0<\varepsilon<1 .
$$

$$
l=\left[m+\frac{1}{2}(n+\varepsilon)+\frac{1}{2}\right] .
$$

I take first the case $n=2 v$. Then

so that

$$
l=m+v
$$

Since

we obtain

$$
2^{\frac{1}{2}} l=2^{\frac{1}{2}} m+2^{\frac{1}{2}} v=2^{\frac{1}{2}} m+2^{-\frac{1}{2}} n=m+\left(2^{\frac{1}{2}}-1\right) m+2^{-\frac{1}{2}} n \text {. }
$$

Since

we have

$$
m>2^{-\frac{1}{2}} n,
$$

$$
2^{\frac{1}{2}} l>m+\left(1-2^{-\frac{1}{2}}\right) n+2^{-\frac{1}{2}} n=m+n \text {. }
$$

$$
2^{\frac{1}{2}} m<n+1 \quad \text { and } \quad 2^{-\frac{1}{2}} n<m,
$$

$$
2^{\frac{1}{2}} l<n+1+2^{\frac{1}{2}} \nu=n+1+2^{-\frac{1}{2}} n<m+n+1
$$

so that in this case

$$
\left[2^{\frac{1}{2}} l\right]=m+n=\left[\left(1+2^{\frac{1}{2}}\right) m\right] .
$$

Then let $n$ be odd $=2 v+1$. We obtain 


$$
l=m+v+1,
$$

whence

$$
\begin{aligned}
2^{\frac{1}{2}} l & =2^{\frac{1}{2}} m+2^{\frac{1}{2}} \frac{1}{2}(n+1)=m+\left(2^{\frac{1}{2}}-1\right) m+2^{-\frac{1}{2}}(n+1) \\
& <m+\left(1-2^{-\frac{1}{2}}\right)(n+1)+2^{-\frac{1}{2}}(n+1)=m+n+1,
\end{aligned}
$$

and on the other hand

$$
2^{\frac{1}{2}} l>m+n
$$

because

$$
2^{\frac{1}{2}} m>n \quad \text { and } \quad 2^{-\frac{1}{2}}(n+1)>m .
$$

Hence in this case as well we have

$$
\left[2^{\frac{1}{2}} l\right]=m+n=\left[\left(1+2^{\frac{1}{2}}\right) m\right] .
$$

It is quite curious to observe that whereas every integer of the form $\left[\left(1+2^{\frac{1}{2}}\right) n\right]$ is also of the form $\left[2^{\frac{1}{2}} n\right]$, no integer of the form $\left[\left(2+2^{\frac{1}{2}}\right) n\right]$ is of the form $\left[2^{\frac{1}{2}} n\right]$. Indeed, the two latter sets of integers, those of form $\left[2^{\frac{1}{2}} n\right]$ and those of form $\left[\left(2+2^{\frac{1}{2}}\right) n\right]$, are two complementary subsets of the natural number series. (See Theorem $3 \mathrm{~b}$ for $l=m=2$ or Theorem 4.)

It is clear that these considerations can be extended in different directions. For example, one might ask if it is possible, also for $m>2$, to find $m$ different irrational numbers $\alpha_{1}, \ldots, \alpha_{m}$, such that

$$
\left[\alpha_{1} n\right], \ldots,\left[\alpha_{m} n\right],
$$

for $n=1,2, \ldots$ in infinitum, furnish $m$ mutually disjoint sets with the whole number series as their union. I shall show below (Section 7) that the answer to this question is negative.

4. Instead of pairs with given differences one might consider triples $\left(a_{n}, b_{n}, c_{n}\right)$ such that the second differences $a_{n}-2 b_{n}+c_{n}$ for $n=1,2, \ldots$ have given values. I mention an example.

Let $\left(a_{1}, b_{1}, c_{1}\right)$ be $(1,2,4)$ so that the second difference here is 1 , and let $a_{n}, b_{n}, c_{n}$ be determined recursively by letting $a_{n}$ be the least integer different from all $a_{r}, b_{r}, c_{r}$, where $r<n, b_{n}$ the least integer different from all $a_{r}, b_{r}, c_{r}$ with $r<n$ and from $a_{n}$, while $c_{n}$ is so chosen that

$$
a_{n}-2 b_{n}+c_{n}=n \text {. }
$$

The first twelve of these triples are

$$
\begin{aligned}
& (1,2,4)(3,5,9)(6,7,11)(8,10,16)(12,13,19)(14,15,22) \\
& (17,18,26)(20,21,30)(23,24,34)(25,27,39)(28,29,41) \\
& (31,32,45) .
\end{aligned}
$$

I have attempted to find a general formula for the $n$th of these triples by the aid of the operation [], buth without success. 
With more success I have treated the triples obtained by the following recursion. Let $a_{1}=1, b_{1}=2, c_{1}=3$. Whenever $a_{r}, b_{r}, c_{r}$ are already determined for $r \leqq n, a_{n+1}$ is chosen as the least integer different from all those $a_{r}, b_{r}, c_{r}$, then $b_{n+1}$ is chosen as the $(n+1)$ th integer different from all the $a_{r}, b_{r}, c_{r}$ and from $a_{n+1}$, while $c_{n+1}$ is put $=b_{n+1}+n+1$. The first eight of these triples are

$$
\begin{gathered}
(1,2,3)(4,6,8)(5,10,13)(7,14,18)(9,17,22) \\
(11,21,27)(12,25,32)(15,29,37)
\end{gathered}
$$

Here I have found the general formulas:

$$
\begin{aligned}
a_{2 n}=\left[\frac{1}{2}\left(3+21^{\frac{1}{2}}\right) n+\frac{1}{6}\left(-3+21^{\frac{1}{2}}\right)\right], & a_{2 n+1}=\left[\frac{1}{2}\left(3+21^{\frac{1}{2}}\right) n+\frac{1}{3} \cdot 21^{\frac{1}{2}}\right] \\
b_{n}=\left[\frac{1}{2}\left(3+21^{\frac{1}{2}}\right) n\right]-1, & c_{n}=\left[\frac{1}{2}\left(3+21^{\frac{1}{2}}\right) n\right]+n-1 .
\end{aligned}
$$

5. My colleague I. Johansson pointed out to me that it could be seen almost immediately that $N_{1}$ and $N_{2}$ are disjoint, $N_{1}$ being the integers [ $\alpha n]$ for integral $n, N_{2}$ the integers $[\beta n]$, if $\alpha$ and $\beta$ are positive irrational numbers such that

$$
\alpha^{-1}+\beta^{-1}=1 .
$$

Indeed, the proof is simply this: Let us assume that integers $m$ and $n$ exist such that $l=[\alpha m]=[\beta n]$. Then we have

or

$$
\begin{aligned}
l<\alpha m<l+1, & l<\beta m<l+1, \\
\alpha^{-1} l<m<\alpha^{-1}(l+1), & \beta^{-1} l<n<\beta^{-1}(l+1),
\end{aligned}
$$

whence by addition, taking into account that $\alpha^{-1}+\beta^{-1}=1$, we obtain $l<m+n<l+1$, which is impossible.

By the way one observes at once that this can be generalized by putting

$$
\alpha^{-1}+\beta^{-1}=c^{-1}
$$

and here supposing only that $c$ is a positive integer. Indeed this assumption leads by the same development to the inequality

$$
l<c(m+n)<l+1
$$

which is impossible in integers $l, m, n, c$.

One might now perhaps be tempted to believe that the last sufficient condition for the non-existence of elements common to $N_{1}$ and $N_{2}$ also is necessary. However, the situation is not so simple, as can be seen from the fact mentioned in Section 3 that all numbers $\left[\left(1+2^{\frac{1}{2}}\right) n\right]$ are also numbers $\left[2^{\frac{1}{2}} n\right]$, whereas no number of the last form is of the form $\left[\left(2+2^{\frac{1}{2}}\right) n\right]:$ The sum 


$$
\frac{1}{1+2^{\frac{1}{2}}}+\frac{1}{2+2^{\frac{1}{2}}}=2^{-\frac{1}{2}}
$$

is not a number of the form 1 divided by an integer. Indeed, a still weaker sufficient condition, which is also necessary, is given in Theorem 8 .

6. Some theorems can suitably be added. In these $N, N_{1}$ and $N_{2}$ retain their earlier meanings.

Theorem 4. If $\alpha^{-1}+\beta^{-1}=1$, then $N_{1}$ and $N_{2}$ are complementary subsets of $N$.

Proof. Since $\alpha$ and $\beta$ shall be $>0$, they are both $>1$. We may also suppose that $1<\alpha<2$. Indeed if $\alpha$ and $\beta$ were both $>2$, we would obtain $\alpha^{-1}+\beta^{-1}<1$. It is then evident that always

$$
[\alpha(n+1)]=[\alpha n]+1 \text { or }[\alpha n]+2 .
$$

In order to prove that every integer is either of the form $[\alpha n]$ or of the form $[\beta m]$ I have to show that when

then

$$
[\alpha(n+1)]=[\alpha n]+2,
$$

$$
[\alpha n]+1=[\beta m]
$$

for a certain integer $m$. Let

$$
\alpha=1+x, \quad 0<x<1,
$$

and let $k$ be a positive integer. Then for $n=[k / x]$

because

$$
[\alpha n]=[n+n \varkappa]=n+[n \varkappa]=n+k-1
$$

so that obviously

$$
n x<k<n x+x<x n+1
$$

On the other hand

$$
[n \varkappa]=k-1 \text {. }
$$

$$
[\alpha(n+1)]=n+1+[(n+1) x]=n+1+k,
$$

since $k<(n+1) x<k+1$. It is clear that, for $k=1,2, \ldots$ we have just the jumps by 2 , which the value of $[\alpha n]$ makes when $n$ increases by 1 . Now, since

$$
\beta=\frac{\alpha}{\alpha-1}=\frac{1+\varkappa}{\varkappa}=1+\frac{1}{\varkappa},
$$

we get

$$
[k \beta]=k+[k / x]=n+k=[\alpha n]+1 \text {. }
$$


Thus, when $[\alpha(n+1)]=[\alpha n]+2$, then the intermediate number $[\alpha n]+1$ has the form $[k \beta]$.

TheOREM 5. If $1, \alpha^{-1}, \beta^{-1}$ are linearly independent (relative to the field of rationals), then $N_{1}$ and $N_{2}$ have an infinite number of common elements.

Proof. It is again clear that we can assume $\alpha$ and $\beta>1$, because if $\alpha<1$, every natural number is of the form $[\alpha n]$. It follows from wellknown theorems in the theory of diophantine approximations that infinitely many triples of integers $l, m, n$ exist such that

whence

$$
-\alpha^{-1}<\alpha^{-1} l-m<0, \quad-\beta^{-1}<\beta^{-1} l-n<0,
$$

so that

$$
l<\alpha m<l+1, \quad l<\beta n<l+1,
$$

$$
[\alpha m]=[\beta n]=l \text {. }
$$

Theorem 6. Let $\alpha$ and $\beta$ be irrational numbers, but $1, \alpha^{-1}, \beta^{-1}$ linearly dependent in such a way that in the equation

$$
a \alpha^{-1}+b \beta^{-1}=c, \quad c>0, \quad a, b, c \text { integers },
$$

$a$ and $b$ have opposite signs. Then $N_{1}$ and $N_{2}$ have an infinite number of common elements.

Proof. For a positive integer $z$ let $x_{z}$ and $y_{z}$ denote the numbers $z \alpha^{-1}, z \beta^{-1}$ reduced modulo 1 , so that $0<x_{z}<1,0<y_{z}<1$. Then the points $\left(x_{z}, y_{z}\right)$ lie on a certain number of straight line segments crossing the unit square. All these segments have the same slope, namely $-a / b$, and one of them ends at the point $(1,1)$. The points $\left(x_{z}, y_{z}\right)$ lie everywhere dense on the segments. Therefore infinitely many $\left(x_{z}, y_{z}\right)$ lie in the region

$$
1-\alpha^{-1}<x<1, \quad 1-\beta^{-1}<y<1,
$$

which leads to the same conclusion as in the case of the preceding theorem.

Theorem 7. If the irrational numbers $\alpha$ and $\beta$ are connected by an equation

$$
a \alpha^{-1}+b \beta^{-1}=c, \quad a>0, b>0, c>1, \quad a, b, c \text { integers },
$$

where the greatest common divisor of $a, b$ and $c$ equals 1 , then $N_{1}$ and $N_{2}$ have infinitely many common elements.

Proof. Just as before, the points $\left(x_{z}, y_{z}\right)$ lie on a number of line segments crossing the unit square, all of them possessing the same slope 
$-a / b$. Since the points $\left(x_{z}, y_{z}\right)$ lie everywhere dense on the segments, it follows that infinitely many of them are in the rectangle

$$
1-\alpha^{-1}<x<1, \quad 1-\beta^{-1}<y<1,
$$

if it is shown that one of the lines enters into this region. This, however, is very easily seen, because either $a \alpha^{-1}>1$ or $b \beta^{-1}>1$, since $c \geqq 2$. Thus we have either $\alpha^{-1}>a^{-1}$ or $\beta^{-1}>b^{-1}$, while the line segment $\lambda$ lying closest to the point $(1,1)$ connects the points $\left(1-a^{-1}, 1\right)$ and $\left(1,1-b^{-1}\right)$. Therefore $\lambda$ must enter the said rectangle.

On the other hand it may be noticed that, if the irrational numbers $\alpha$ and $\beta$ are connected by the equation

$$
a \alpha^{-1}+b \beta^{-1}=1,
$$

where the integers $a$ and $b$ are $>0$, then $N_{1}$ and $N_{2}$ are disjoint. This is seen by the fact that $N_{1}$ is contained in the set of all $\left[\alpha a^{-1} n\right]$ and $N_{2}$ contained in the set of all $\left[\beta b^{-1} n\right]$; for in Section 5 we have remarked that the two sets $\left[\alpha_{1} n\right]$ and $\left[\beta_{1} n\right]$ are disjoint when

From all this follows

$$
\alpha_{1}^{-1}+\beta_{1}^{-1}=1 \text {. }
$$

Theorem 8. A necessary and sufficient condition for the sets [an] and $[\beta n]$ to be disjoint is that $\alpha$ and $\beta$ are connected by an equation

$$
a \alpha^{-1}+b \beta^{-1}=1,
$$

where $a$ and $b$ are positive integers.

7. As an application we may prove the nonexistence of 3 irrationals $\alpha, \beta, \gamma$ such that the corresponding sets $N_{1}, N_{2}, N_{3}$ are mutually disjoint ( $N_{1}$ being the set of all [ $\left.\alpha n\right]$, etc.). Indeed, if $\alpha, \beta, \gamma$ should possess this property, it follows from Theorem 8 that we should have 3 equations

$$
a_{1} \alpha^{-1}+b_{1} \beta^{-1}=1, \quad a_{2} \alpha^{-1}+b_{2} \gamma^{-1}=1, \quad a_{3} \beta^{-1}+b_{3} \gamma^{-1}=1
$$

with positive coefficients $a_{i}$ and $b_{i}, i=1,2,3$. The elimination of $\gamma$ between the two last equations yields

$$
a_{2} b_{3} \alpha^{-1}-a_{3} b_{2} \beta^{-1}=b_{3}-b_{2},
$$

where the coefficients have not all the same sign. Therefore the last equation is independent of $a_{1} \alpha^{-1}+b_{1} \beta^{-1}=1$ so that $\alpha, \beta, \gamma$ must all be rational.

If the operation of taking the greatest integer $\leqq \xi$ is iterated, we may 
of course get expressions furnishing an arbitrary number of disjoint sets of integers. For example, the three expressions

$$
[\alpha[\alpha n]], \quad\left[\alpha\left[\alpha^{2} n\right]\right], \quad\left[\alpha^{2} n\right],
$$

where $\alpha=\frac{1}{2}\left(1+5^{\frac{1}{2}}\right)$, yields three subsets of $N$ which are mutually disjoint and have $N$ as their union.

8. The theorems in the present paper concerning the sets $[\alpha n]$ are extended to the more general sets of the form $[\alpha n+\beta]$ in a paper to appear in Norske Vid. Selsk. Forh., Trondheim.

See also the following paper by Bang [1].

\section{REFERENCES}

1. Th. Bang, On the sequence $[n \alpha], n=1,2, \ldots$ Supplementary note to the preceding paper by Th. Skolem, Math. Scand. 5 (1957), 69-76.

2. J. Steiner, Combinatorische Aufgabe, J. reine angew. Math. 45 (1853), 181-182 (=Gesammelte Werke II, Berlin, 1882, 437-438). 